\title{
Investigation of bacterial and fungal diversity in tarag using high-throughput sequencing
}

\author{
Zhihong Sun, ${ }^{*} \dagger^{1}$ Wenjun Liu, ${ }^{*} \dagger^{1}$ Qiuhua Bao, ${ }^{*}$ Jiachao Zhang, ${ }^{*}$ Qiangchuan Hou, ${ }^{,}$Laiyu Kwok, ${ }^{*}$ \\ Tiansong Sun, ${ }^{*}$ and Heping Zhang* ${ }^{2}$ \\ *Key Laboratory of Dairy Biotechnology and Engineering, Ministry of Education; Dairy Processing Laboratory of National Dairy Production \\ Technology and Research Center, Ministry of Agriculture, Inner Mongolia Agricultural University, Hohhot, Inner Mongolia, 010018, China \\ †Synergetic Innovation Center of Food Safety and Nutrition, Northeast Agricultural University, Harbin, Heilongjiang, 150030, China
}

\begin{abstract}
This is the first study on the bacterial and fungal community diversity in 17 tarag samples (naturally fermented dairy products) through a metagenomic approach involving high-throughput pyrosequencing. Our results revealed the presence of a total of 47 bacterial and 43 fungal genera in all tarag samples, in which Lactobacillus and Galactomyces were the predominant genera of bacteria and fungi, respectively. The number of some microbial genera, such as Lactococcus, Acetobacter, Saccharomyces, Trichosporon, and Kluyveromyces, among others, was found to vary between different samples. Altogether, our results showed that the microbial flora in different samples may be stratified by geographic region.
\end{abstract}

Key words: tarag, pyrosequencing, bacterial diversity, fungal diversity

\section{INTRODUCTION}

In northeast Asia, lives of nomadic people greatly depend on the domestic livestock such as horses, cows, yaks, goats, ewes, and camels, and they have produced various kinds of naturally fermented dairy products from the milk of these animals (Shuangquan et al., 2006; Sun et al., 2010b; Yu et al., 2011; Bao et al., 2012b; Liu et al., 2012a). Tarag, fermented milk of cows, is a special naturally fermented dairy product in Mongolia and the northwest of China. The history of making and drinking tarag in these regions dates back several thousand years (Airidengcaicike et al., 2010). Tarag was generally made by natural fermentation without the use of any commercial starter cultures, and the natural cultures were subsequently used to inoculate fresh milk in the next day. To produce acidity, alcohol, and flavor to the desired level, tarag would be fermented for at least 5 to

Received May 14, 2014.

Accepted June 28, 2014

${ }^{1}$ These authors contributed equally to this work.

${ }^{2}$ Corresponding author: hepingdd@vip.sina.com
$8 \mathrm{~d}$ at ambient temperature around 10 to $25^{\circ} \mathrm{C}$. In this way, a stable microbial phase was naturally formed, preserved, and handed down through generations together with the traditional tarag.

The naturally fermented dairy products, such as koumiss and tarag, have been considered not only as a kind of food, but also a complete nutriment with medicinal properties (Ishii and Konagaya, 2002). In China and Mongolia, nomads created the "koumiss therapy," which combined traditional medicine with koumiss consumption to help in the treatment of hepatitis, chronic ulcer, tuberculosis, and so on (Hasisurong et al., 2003). Before we are able to understand the mechanism of such kinds of folk therapies, an in-depth characterization of the microbial communities in tarag is necessary to provide valuable information. It has been reported that lactic acid bacteria and yeasts play key roles during tarag production. By conventional culture and molecularbiology methods, Watanabe et al. (2008) reported that Lactobacillus, Streptococcus, and alcoholic-fermenting yeasts such as Kluyveromyces and Saccharomyces were the predominant microbial species in tarag.

Recently, more and more molecular technology based on culture-independent methods has been applied to the evaluation of microbial diversity in different fermented dairy products, namely, cheese (El-Baradei et al., 2007; Van Hoorde et al., 2008), tarag (Liu et al., 2012b), and kefir (Chen et al., 2008). Although these methods, such as denaturing gradient gel electrophoresis (Chen et al., 2008; Van Hoorde et al., 2008), temporal temperature gel electrophoresis (El-Baradei et al., 2007), and Sanger sequencing of $16 \mathrm{~S}$ rRNA gene clone library (Liu et al., $2012 \mathrm{~b}$ ) have been used to investigate the microbial diversity, they may provide an inaccurate description of the total microbial structure.

Pyrosequencing, a next-generation DNA sequencing platform, based on the detection of pyrophosphate released during nucleotide incorporation, was developed by Margulies et al. (2005). Recently, this technique has successfully been used to detect the microbial communities in various fermented foods, such as cheese (Masoud 
et al., 2011; Alegría et al., 2012; Quigley et al., 2012), fermented seafood (Roh et al., 2010; Jung et al., 2013), fermented soybeans (Nam et al., 2012), pearl millet slurries (Humblot and Guyot, 2009), kefir (Dobson et al., 2011; Leite et al., 2012), fermented mung beans (Chao et al., 2013), and sourdoughs (Lattanzi et al., 2013). However, to our knowledge, this approach has, so far, not been applied in the investigation of microbial diversity in tarag. Meanwhile, the diversity of yeast in tarag has never been studied using culture-independent methods.

Therefore, we intended to investigate the microbial communities of the bacterial and fungal diversity in tarag, which would provide new insights into the microbiomes of tarag at the metagenomic level. In this study, the bacterial and fungal diversities in 17 tarag samples were evaluated with 454 pyrosequencing, and the microbial phase was systematically analyzed.

\section{MATERIALS AND METHODS}

\section{Sample Collection}

Seventeen tarag samples were collected from different regions of Mongolia and China. Detailed information of sampling location is listed in Table 1. Samples were aseptically collected and kept in ice boxes during transportation.

\section{Extraction of Microbial DNA and PCR Amplification}

Microbial DNA extraction was carried out according to the protocol of Liu et al. (2012b). The quality of the extracted DNA was assessed by $0.8 \%$ agarose gel electrophoresis, and the ratio of absorbance at 260 and $280 \mathrm{~nm}$ [optical density (OD) 260/280] was measured by spectrophotometry. All of the DNA samples were stored at $-20^{\circ} \mathrm{C}$ until further processing.

A 195-nucleotide sequence of the V3 region of the $16 \mathrm{~S}$ rRNA gene was amplified by PCR for bar-coded pyrosequencing. A set of 6-nucleotide barcodes was added to the universal forward primer 338F (5'-ACTCCTACGGGAGGCAGCAG- $3^{\prime}$ ) and the reverse primer 533R (5'-TTACCGCGGCTGCTGGCAC-3'), which targeted the domain Bacteria (Huse et al., 2008). The internal transcribed spacer (ITS) 1 region of fungi was selected and amplified using the forward primer ITS1F (5'-CTTGGTCATTTAGAGGAAGTAA-3') and the reverse primer ITS2 (5'-GCTGCGTTCTTCATCGATGC-3), to which another set of 6-nucleotide barcodes was added (Ghannoum et al., 2010). Polymerase chain reaction amplification of $16 \mathrm{~S}$ rRNA V3 and ITS regions were performed as described in Humblot and Guyot (2009), and Ghannoum et al. (2010), respectively.

\section{Amplicon Purification, Quantification, Pooling, and Pyrosequencing}

Amplicons generated from PCR were purified using the AMPure XP purification system (Beckman Coulter Genomics, Takeley, UK), and the quality of the purified DNA was checked using an Agilent 2100 Bioanalyzer (Agilent Technologies Inc., Palo Alto, CA). The quantity of amplicons was estimated using an Agilent DNA 1000 kit (Agilent Technologies Inc.). The PCR products were pooled in equimolar ratios to a final concentration of $100 \mathrm{nmol} / \mathrm{L}$ for pyrosequencing using a Roche GS FLX System (F. Hoffmann-La Roche Ltd., Basel, Switzerland) in the Advanced Studies in Genomics, Proteomics and Bioinformatics Laboratory, University of Hawaii (Honolulu).

\section{Sequence Processing and Bioinformatics Analysis}

Sequences generated from pyrosequencing were mainly analyzed with the QIIME (v1.5.0-dev; http:// qiime.org/) suite of software tools for quality adjustment, barcode split, identification of the operational taxonomic units (OTU), taxonomic assignment, community comparison, and statistical analysis (Caporaso et al., 2010b; Kuczynski et al., 2011). Sequences were filtered to minimize the effects of poor sequence quality and sequencing errors by removing sequences with more than 1 ambiguous base calls and retaining only sequences that were $\geq 140$ nucleotides. The extracted sequences were sorted into different samples according to the barcodes.

After the removal of the barcodes and primer, the extracted sequences were aligned using PyNAST software (Caporaso et al., 2010a) and the passed sequences were clustered based on a $97 \%$ sequence identity using the UCLUST algorithm (Edgar, 2010) to obtain a unique V3 and ITS sequence set, respectively. The taxonomy of each bacterial OTU representative sequence was assigned with the Ribosomal Database Project (RDP) II database using RDP classifier with an $80 \%$ confidence threshold (Wang et al., 2007). The representative fungal OTU sequences were phylogenetically classified by standalone Mega BLAST searches of the UNITE database (Abarenkov et al., 2010), a fungal ITS sequence database. Two de novo taxonomic trees were constructed using bacterial and fungal OTU representative sets using FastTree software (Price et al., 2009) for the downstream analysis, including the calculation of the $\alpha$ and $\beta$ diversity.

The Shannon-Wiener index and Chao1 index were used to calculate the $\alpha$ diversity, and the UniFrac metric (Lozupone and Knight, 2005) was used to calculate the $\beta$ diversity both in a weighted and unweighted fashion 
BACTERIAL AND FUNGAL DIVERSITY IN TARAG

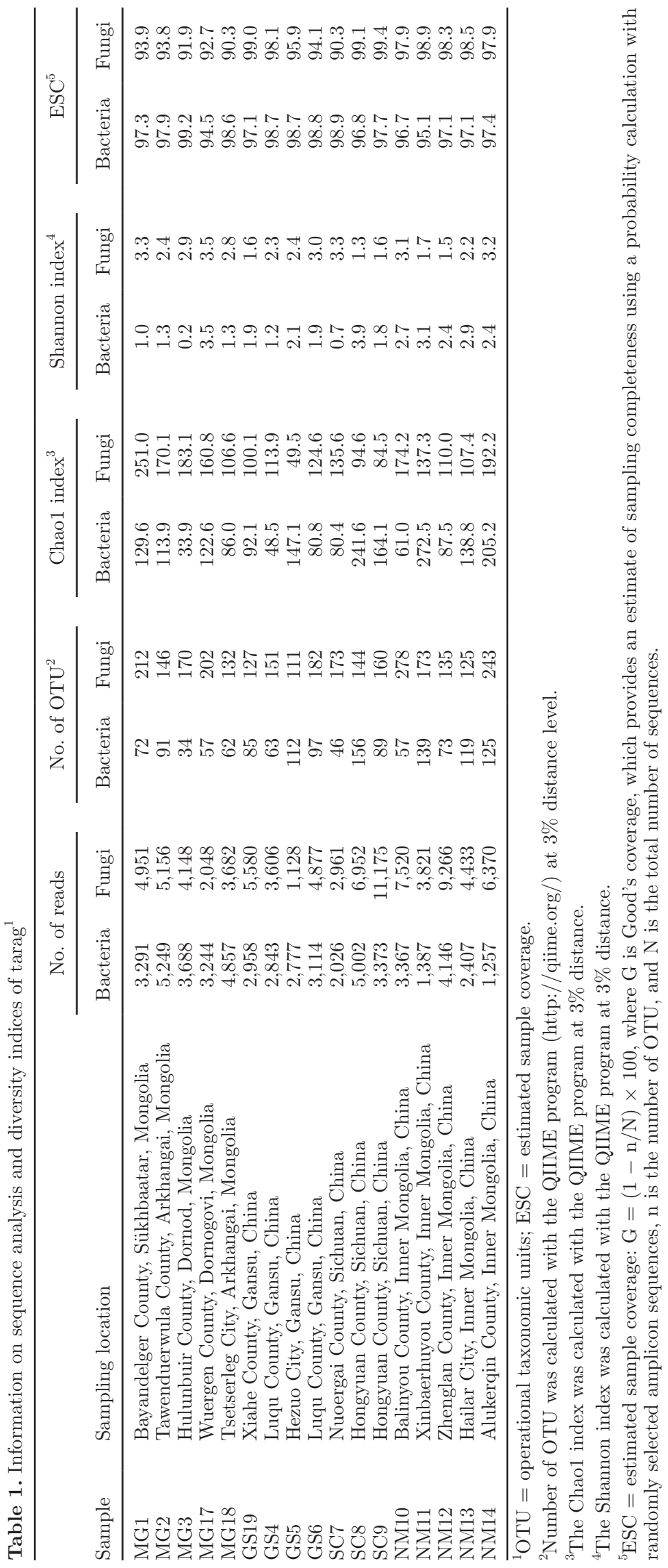




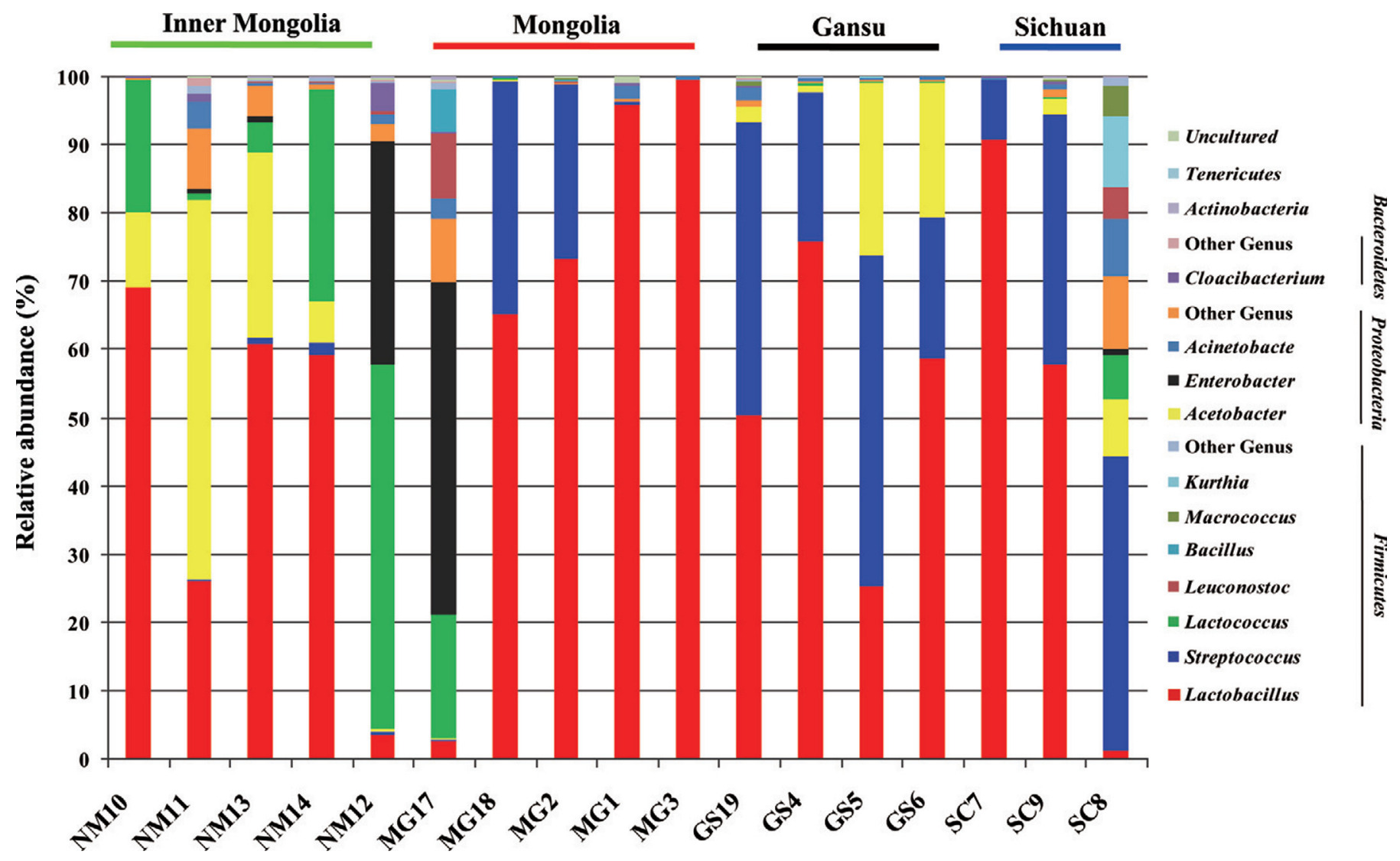

Figure 1. Relative abundance of bacterial sequence read in each tarag sample collected in China and Mongolia.

for the principal components analysis and unweighted pair group method with arithmetic mean clustering. Phylogenetic trees of Lactobacillus OTU representative sequences were constructed and drawn using MAGE 5.0 (Tamura et al., 2011) and Interactive Tree of Life (iTOL; Letunic and Bork, 2007) software, respectively.

\section{Statistical Analysis}

The Mann-Whitney and Kruskal-Wallis tests were used to evaluate the differences in the bacterial and fungal populations. Multivariate ANOVA (MANOVA) and clustering of microbiota of tarag based on Mahalanobis distances calculated using MANOVA were conducted in Matlab R2011b software (The MathWorks Inc., Natick, MA).

\section{Nucleotide Sequence Accession Numbers}

The sequence data reported in this paper have been deposited in the MG-RAST database (http://metagenomics.anl.gov/; accession no. 2912, 4509270-4509296).

\section{RESULTS AND DISCUSSION}

\section{Richness and Diversity Analysis}

After preprocessing, a total of 142,660 high-quality reads were obtained from the one-fourth plate using a 454 genome sequencer (454 Life Sciences, Branford, $\mathrm{CT}$ ), corresponding to 54,986 for bacteria and 87,674 reads for fungi. Average read length was 155 bases for bacteria and 180 bases for fungi, and each individual sample was covered by an average of $3,234( \pm 1,140)$ and $5,157( \pm 2,528)$ bacterial and fungal reads, respectively. The average number of OTU for bacteria and fungi was $87( \pm 34)$ and $168( \pm 44)$ at $3 \%$ sequence dissimilarities, respectively (Table 1 ).

Although the individual rarefaction curves for the bacterial and fungal diversity for most samples plateaued with the current sequencing, they failed to reach the saturation phase (Supplemental Figure S1; http:// dx.doi.org/10.3168/jds.2014-8360). The Shannon diversity estimates of the samples did reach stable values (Supplemental Figure S2). These results indicate that although new phylotypes would be expected with ad- 
ditional sequencing, most of the microbial diversity had already been captured (Wang et al., 2012).

In addition, the Good's coverage, an estimator of completeness of sampling, highlighted good overall sampling with levels of 94.5 to $99.2 \%$ and 90.3 to $99.4 \%$ for bacteria and fungi, respectively. These results indicated that although new phylotypes would be expected with additional sequencing, the majority of bacterial and fungal phylotypes present in tarag had already been captured (Jung et al., 2012).

We compared the microbial diversity, as estimated by the Shannon index, and the results showed that the index of bacteria $(1.46 \pm 1.23)$ was significantly lower than that of fungi $(2.98 \pm 0.43)$ in tarag collected in Mongolia $(P<0.05)$. Similarly, the observed number of bacterial OTU $(87 \pm 34)$ was significantly lower than that of fungi $(168 \pm 44 ; P<0.05)$. However, the difference between the Shannon index of bacteria and fungi was not statistically significant $(P>0.05)$ in tarag collected in Inner Mongolia, Sichuan, and Gansu (China). These indicated more phylotypes of fungi than bacteria existing in tarag collected in Mongolia, but not samples from the studied Chinese provinces. In contrast, Wata- nabe et al. (2008) and Sun et al. (2010b) reported that the number of isolated strains and concentration of lactic acid bacteria were significantly higher than yeasts in tarag, a traditionally fermented dairy product of Mongolia. This contradiction may be due to differences in the analytical goals and methods used.

\section{Taxonomic Profiling of Bacterial Communities}

The phylogenetic classification of bacterial sequences from tarag is summarized in Figure 1. The sequences were distributed among 4 bacterial phyla, namely Firmicutes, Proteobacteria, Bacteroidetes, and Actinobacteria. Of the 4 bacterial phyla, Firmicutes dominated in all tarag samples from different sampling locations, containing 213 OTU, corresponding to 86.40, 86.54, 88.64, and $66.83 \%$ of the reads from Mongolia, and Gansu, Sichuan, and Inner Mongolia provinces, respectively. The second dominated bacterial phylum, comprising 192 OTU, was Proteobacteria, representing an average of $17.15 \%( \pm 9.63 \%)$ of the population. Bacteroidetes $(0.53 \pm 0.59 \%)$, Actinobacteria (0.11 \pm $0.05 \%)$, and unclassified bacteria $(0.11 \pm 0.07 \%)$ were

Table 2. Percentage of bacterial sequence reads at the phylum and genus level in tarag samples from Mongolia and 3 provinces in China

\begin{tabular}{|c|c|c|c|c|}
\hline \multirow[b]{2}{*}{ Bacteria } & \multicolumn{4}{|c|}{ Region } \\
\hline & $\begin{array}{l}\text { Mongolia } \\
(\mathrm{n}=5)\end{array}$ & $\begin{array}{c}\text { Gansu } \\
(\mathrm{n}=4)\end{array}$ & $\begin{array}{l}\text { Sichuan } \\
(\mathrm{n}=3)\end{array}$ & $\begin{array}{l}\text { Inner Mongolia } \\
\quad(\mathrm{n}=5)\end{array}$ \\
\hline \multicolumn{5}{|l|}{ Phylum } \\
\hline Firmicutes & 86.40 & 86.54 & 88.64 & 66.83 \\
\hline Proteobacteria & 13.01 & 13.19 & 10.87 & 31.51 \\
\hline Bacteroidetes & 0.25 & 0.19 & 0.26 & 1.41 \\
\hline Actinobacteria & 0.12 & 0.04 & 0.15 & 0.13 \\
\hline Uncultured & 0.21 & 0.04 & 0.08 & 0.12 \\
\hline \multicolumn{5}{|l|}{ Genus } \\
\hline Lactobacillus & 67.22 & 52.51 & 49.91 & 43.70 \\
\hline Streptococcus & 12.07 & 33.55 & 29.51 & 0.71 \\
\hline Lactococcus & 3.66 & 0.17 & 2.26 & 21.86 \\
\hline Leuconostoc & 1.92 & 0.02 & 1.60 & 0.16 \\
\hline Acinetobacter & 1.13 & 0.75 & 3.09 & 1.31 \\
\hline Enhydrobacter & 0.15 & 0.16 & 0.42 & 0.37 \\
\hline Cloacibacterium & 0.15 & 0.09 & 0.18 & 1.09 \\
\hline Acetobacter & 0.10 & 12.09 & 3.57 & 20.06 \\
\hline Macrococcus & 0.05 & 0.17 & 1.52 & 0.02 \\
\hline Chryseobacterium & 0.05 & 0.03 & 0.02 & 0.12 \\
\hline Delftia & 0.03 & 0.01 & 0.03 & 0.12 \\
\hline Escherichia & 0.02 & 0.05 & 0.06 & 0.04 \\
\hline Rhizobium & 0.02 & 0.01 & 0.16 & 0.09 \\
\hline Roseomonas & 0.02 & 0.01 & 0.03 & 0.06 \\
\hline Kurthia & 0.01 & 0.02 & 3.49 & 0 \\
\hline Enterobacter & 9.77 & 0 & 0.25 & 6.86 \\
\hline Klebsiella & 1.37 & 0 & 0.01 & 0.13 \\
\hline Bacillus & 1.30 & 0 & 0.02 & 0 \\
\hline Citrobacter & 0.14 & 0 & 0.05 & 0.19 \\
\hline Bifidobacterium & 0.10 & 0 & 0.01 & 0.04 \\
\hline Other & 0.72 & 0.36 & 3.81 & 3.07 \\
\hline
\end{tabular}


Table 3. Percentage of fungal sequence reads at the phylum and genus level in tarag samples from Mongolia and 3 provinces in China

\begin{tabular}{lcccc}
\hline & & & Region & \\
\cline { 2 - 4 } Fungi & $\begin{array}{c}\text { Mongolia } \\
(\mathrm{n}=5)\end{array}$ & $\begin{array}{c}\text { Gansu } \\
(\mathrm{n}=4)\end{array}$ & $\begin{array}{c}\text { Sichuan } \\
(\mathrm{n}=3)\end{array}$ & $\begin{array}{c}\text { Inner Mongolia } \\
(\mathrm{n}=5)\end{array}$ \\
\hline Phylum & & & & \\
Ascomycota & 96.87 & 98.78 & 98.71 & 73.76 \\
Basidiomycota & 2.68 & 1.06 & 1.05 & 26.02 \\
Mucoromycotina & 0.01 & 0 & 0.07 & 0 \\
Uncultured & 0.44 & 0.18 & 0.17 & 0.22 \\
Genus & & & & \\
Galactomyces & 15.72 & 58.63 & 75.52 & 0.43 \\
Saccharomyces & 60.40 & 19.66 & 19.23 & 25.66 \\
Trichosporon & 0.58 & 0.20 & 0.51 & 4.52 \\
Kluyveromyces & 9.97 & 10.4 & 0.01 & 3.54 \\
Pichia & 2.12 & 7.96 & 0.49 & 0.01 \\
Candida & 0.75 & 0.51 & 2.30 & 0.04 \\
Torulaspora & 4.72 & 0.19 & 0.38 & 0.07 \\
Debaryomyces & 0.41 & 0.99 & 0.57 & 0.13 \\
Cryptococcus & 0.63 & 0.14 & 0.11 & 0.06 \\
Rhodotorula & 0.46 & 0.04 & 0.20 & 0.04 \\
Guehomyces & 0.05 & 0.61 & 0.08 & 1.68 \\
Cladosporium & 0.14 & 0.33 & 0.10 & 5.64 \\
Kazachstania & 1.09 & 0.03 & 0 & 0.50 \\
Other & 2.96 & 0.31 & & \\
\hline
\end{tabular}

the minor members of the domain bacteria in all tarag samples (Table 2). These results were in agreement with some previous studies that confirmed the presence of the 4 major bacterial phyla in the fermented foods, such as fermented seafood (Roh et al., 2010), pearl millet slurries (Humblot and Guyot, 2009), kefir (Dobson et al., 2011), alcoholic beverages (Jung et al., 2012), kochujang (Nam et al., 2012), and artisanal cheeses (Quigley et al., 2012), and Firmicutes was the predominant bacterial phylum. However, different results were reported in the previous studies described by Leite et al. (2012) and Illeghems et al. (2012), which only found 3 major phyla, including Firmicutes, Proteobacteria, and Actinobacteria, in kefir and cocoa bean. The latter results indicated that Firmicutes was the predominant bacterial phylum in the environment of those fermented foods.

At the genus level, a total of 47 bacterial genera were identified and distributed among the 4 phyla, dominated by Lactobacillus. Further analysis revealed that 14 genera were found to be common in all tarag samples [from 4 different sampling locations (Mongolia, and Gansu, Sichuan, and Inner Mongolia provinces)]: Lactobacillus (67.22, 52.51, 49.91, and 43.70\%), Streptococcus (12.07, $33.55,29.51$, and $0.71 \%$ ), Lactococcus $(3.66,0.17,2.26$, and $21.86 \%)$, Leuconostoc $(1.92,0.02,1.60$, and $0.16 \%)$, Acinetobacter (1.13, 0.75, 3.09, and 1.31\%), Enhydrobacter $(0.15,0.16,0.42$, and $0.37 \%)$, Cloacibacterium $(0.15,0.09,0.18$, and $1.09 \%)$, Acetobacter $(0.10,12.09$, 3.57 , and 20.06\%), Macrococcus $(0.05,0.17,1.52$, and
$0.02 \%)$, Chryseobacterium (0.05, 0.03, 0.02, and $0.12 \%)$, Delftia $(0.03,0.01,0.03$, and $0.12 \%)$, Escherichia $(0.02$, $0.05,0.06$, and $0.04 \%)$, Rhizobium $(0.02,0.01,0.16$, and $0.09 \%)$, and Roseomonas $(0.02,0.01,0.03$, and $0.06 \%$ ). It is well known that Lactobacillus and Streptococcus are indeed 2 widespread genera associated with tarag fermentation processes (Watanabe et al., 2008; Sun et al., 2010a, Bao et al., 2012b). As lactobacilli have been reported to have higher acid tolerance than streptococci and lactococci (Rogosa et al., 1951), the members of the genus of Lactobacillus may be supplied from old tarag that was used as the starter for fermentation. In some previous reports, some species of Lactococcus and Leuconostoc were isolated from raw milk (An et al., 2004; Bao et al., 2012a), which indicated that Lactococcus and Leuconostoc spp. could have been sourced from the raw milk. They were possibly able to grow during the early stage of fermentation, but could not survive in the final products because of their low acid tolerance (Miyamoto et al., 2010). Therefore, our result of the relatively low percentage of Lactococcus and Leuconostoc spp. compared with Lactobacillus and Streptococcus spp. could most likely be explained by the acid inhibition of the 2 former genera during the later stage of fermentation of tarag.

In the current study, 21 genera of Proteobacteria were detected in the different tarag samples, including Acinetobacter, Klebsiella, Escherichia, and Salmonella. This is the first report of the presence of these 4 genera in tarag. The dominating genus was Acetobacter (Table 
3). It is known that Acetobacter spp. have great ability to oxidize ethanol to acetic acid because of their possession of the enzyme alcohol dehydrogenase (Yoo et al., 1994). Therefore, they can produce large amounts of acetic acid from the alcohol produced by the yeasts present in tarag. Escalante et al. (2008) previously reported that the proportion of Proteobacteria, such as Acinetobacter spp., Enterobacter spp., and Escherichia spp., gradually decreased throughout the fermentation process of Mexican alcoholic beverages. In our results, we speculated that these genera were contaminants from the raw milk and, consistent with Escalante et al. (2008), they were subsequently killed during the fermentation process.
For the genus of Lactobacillus, a total of 126 OTU were detected in all tarag samples, in which Lactobacillus delbrueckii ssp. bulgaricus was the predominant species (Figure 2). In all of the OTU, part 1 was identified as the Lactobacillus kefiranofaciens and Lactobacillus helveticus group, containing 78 OTU $(5.63 \%$ of reads for Lactobacillus). The second part, comprising $91.72 \%$ (31 OTU) of the total reads for Lactobacillus, was identified as the Lactobacillus delbrueckii ssp. bulgaricus group. The third part, which contained 17 OTU $(2.65 \%$ of reads for Lactobacillus), was assigned to the Lactobacillus casei and Lactobacillus plantarum group. This result was in agreement with a previous study in which Lactobacillus delbrueckii ssp. bulgaricus was found to be

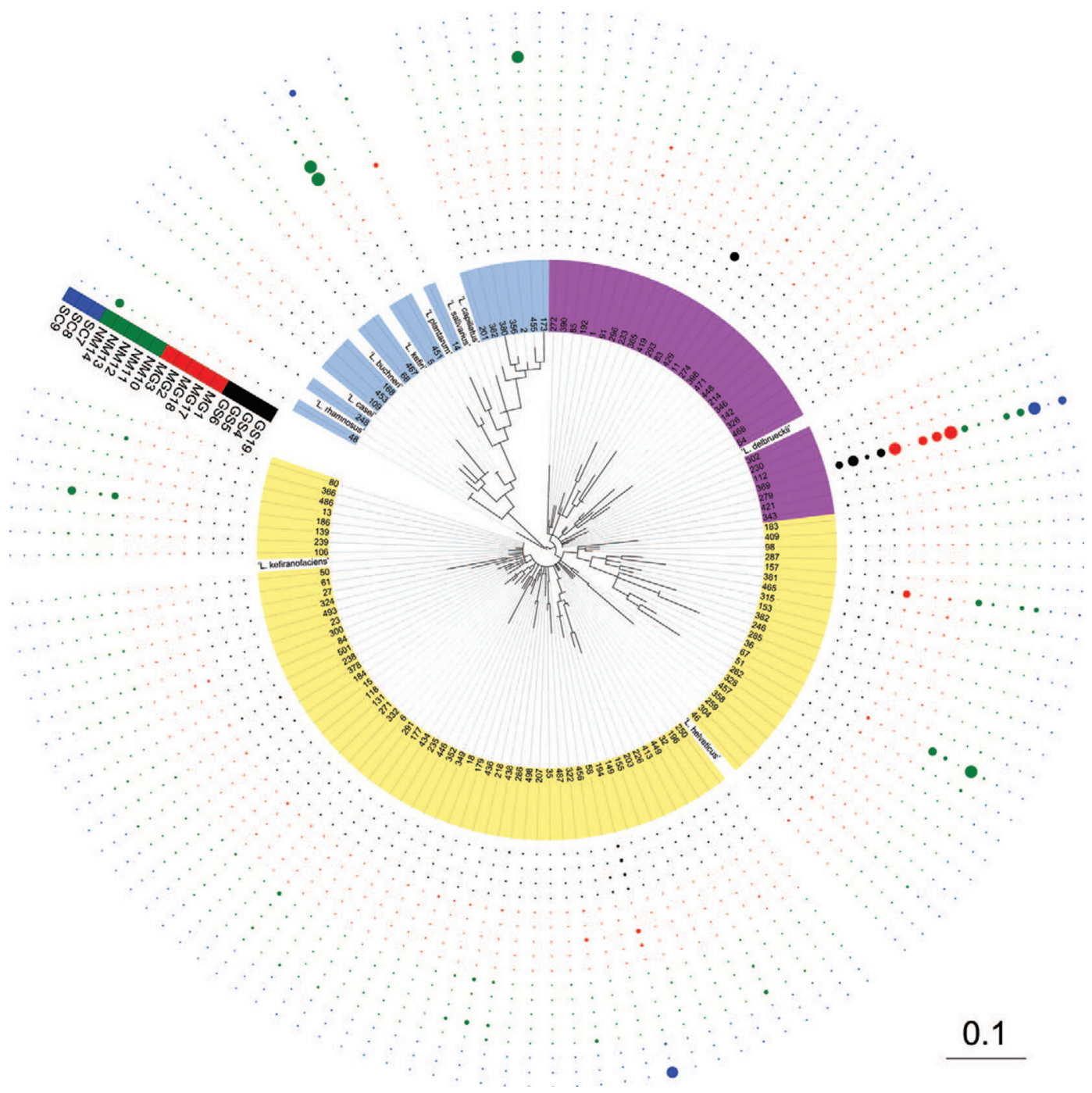

Figure 2. Maximum likelihood tree of all representative Lactobacillus operational taxonomic units (OTU). Sequence labels of representative OTU generated from the current study are colored, whereas sequences retrieved from the GenBank database (http://www.ncbi.nlm.nih.gov/genbank/) are designated with uncolored sequence labels. Each color dot represents an OTU. The color of dot distinguishes the different sampling sites, for which black, red, green, and blue signifies tarag sampled in Gansu (China), Mongolia, Inner Mongolia (China), and Sichuan (China), respectively. The size of dot indicates the abundance of sequence reads. 


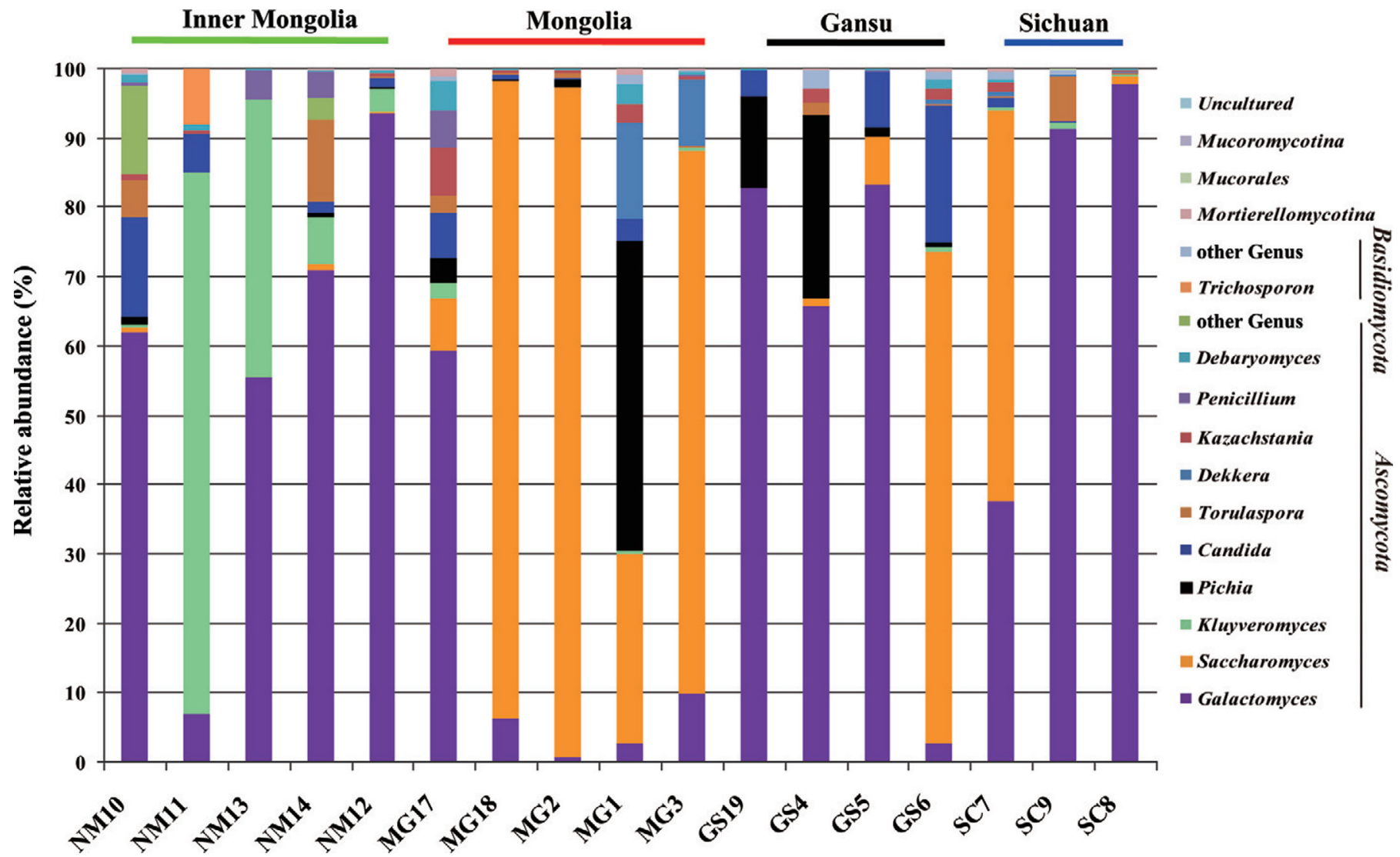

Figure 3. Relative abundance of fungal sequence read in each tarag sample collected in China and Mongolia.

the predominant species of Lactobacillus in tarag from Mongolia (Yu et al., 2011). However, different results were reported in some previous studies of Liu et al. (2012a) and Bao et al. (2012a,b), in which Lactobacillus helveticus was found to be the predominant specie in tarag from Inner Mongolia, Sichuan, and Gansu provinces, respectively. The major reason for this difference could be ascribed to the different methods of analysis (Watanabe et al., 2008). In addition, we compared the content of reads for each OTU (Figure 2). The results indicated that the Lactobacillus casei and Lactobacillus plantarum group was more abundant in the tarag from Inner Mongolia, whereas the Lactobacillus kefiranofaciens and Lactobacillus helveticus group was barely present in the tarag from Mongolia. This leads to the conclusion that different geographic regions may be inhabited with different microbial diversities (Watanabe et al., 2008).

\section{Taxonomic Profiling of the Fungal Community}

The composition of the fungal communities in all tarag samples was simpler than that of the bacterial communities (Figure 3) and contained 2 phyla: Asco- mycota and Basidiomycota, and 1 subphylum: Mucoromycotina. All of the tarag samples were predominated by the phylum Ascomycota, containing 475 OTU and constituting $92.03 \%( \pm 12.21 \%)$ of the fungal populations with the minor members of Basidiomycota $(7.7 \pm$ $12.24 \%)$, Mucoromycotina (0.02 $\pm 0.03 \%)$, and uncultured fungi ( $0.25 \pm 0.13 \%$; Table 3). Similar findings of a predomination of the fungal phylum Ascomycota was reported in Korean alcoholic beverages (Jung et al., 2012), cocoa bean (Illeghems et al., 2012), and Chinese liquor ( $\mathrm{Li}$ et al., 2011). These results indicate that the phylum Ascomycota is the primary fungi in traditional fermented foods.

Likewise, we also carried out an analysis of the fungal composition at the genus level, in which 43 fungal genera were identified. The results showed that the most prevailing fungal genus was Galactomyces, followed by Saccharomyces, Trichosporon, Kluyveromyces, Pichia, and Candida, which accounted for $51.9 \pm 25.47,24.93$ $\pm 25.29,6.74 \pm 12.62,5.21 \pm 5.76,3.77 \pm 3.25$, and $1.78 \pm 1.42 \%$ of all fungal populations, respectively. Although several species of Galactomyces seem to have been rarely reported (Smith et al., 1995), the genus Galactomyces was previously found to be the dominant 
yeast in cheese (Eliskases-Lechner and Ginzinger, 1995; Prillinger et al., 1999). Our data also showed that this genus of fungi was present in high abundance in tarag. The fungi may play a role in lactic acid utilization, lipolysis, proteolysis and flavor development (Wyder et al., 1999). Other significantly present genera, including Saccharomyces, Kluyveromyces, Pichia, and Can-
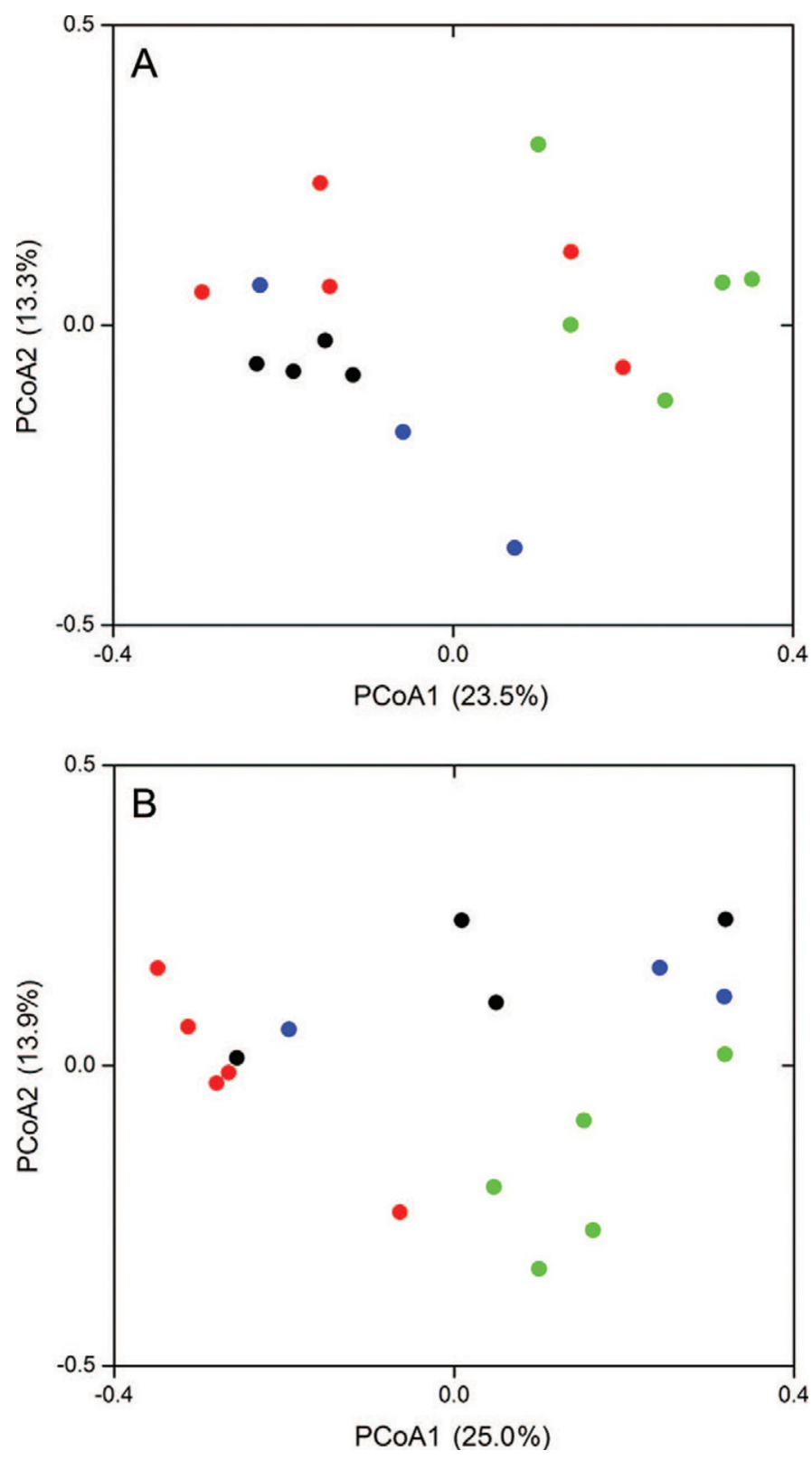

Figure 4. Principal components analysis $(\mathrm{PCoA})$ of the bacterial (A) and fungal (B) diversity in tarag from different sampling sites. The PCoA plots were generated using unweighted UniFrac distances (Lozupone and Knight, 2005) for the operational taxonomic units from samples of different regions. The green, blue, and black symbols represent samples from Inner Mongolia, Sichuan, and Gansu in China, respectively, and the red symbol represents Mongolian tarag. dida, are commonly associated with the fermentation processes of tarag and cheese (Watanabe et al., 2008; Rahman et al., 2009; Miyamoto et al., 2010). They play important roles in processes such as hydrolyzing milk proteins and fat, assimilating lactic acid, and producing ethanol (Sudun et al., 2013). Furthermore, some fungal genera that were not yet reported to be associated with tarag fermentations were detected in the present study. For instance, Trichosporon, a genus of the phylum Basidiomycota, is common in raw milk, which, in a previous study, caused the formation of a surface film on the cottage cheese and led to spoilage (Welthagen and Viljoen, 1998). Our results showed that the percentage of Trichosporon $(25.66 \%)$ was apparently higher in tarag in Inner Mongolia than the other 3 sampling sites. This could indicate poor hygiene and ineffective cleaning procedures, and, hence, reveal the need for improved sanitization procedures in tarag production in Inner Mongolia.

\section{Comparison of Bacterial and Fungal Communities of Tarag}

Multivariate statistical analyses were performed to compare the overall structure of microbiota of all samples. Principal components analysis based on the unweighted UniFrac metric revealed the bacterial microbial communities of the Sichuan and Gansu samples were distinct from those of the Inner Mongolia samples based on the principal components 1 and 2 (23.55 and $13.33 \%$ of variance, respectively), and also revealed that few of the samples in the Mongolian and Inner Mongolia groups overlapped (Figure 4). A MANOVA also indicated that the 4 groups were first separated as 2 clades (Figure 5), with a much smaller within-theclade distance between Mongolian and Inner Mongolia samples compared with Sichuan samples. Principal components analysis also revealed that the fungal communities of Sichuan were distinct from those of Inner Mongolia and Mongolia (principal components 1 and 2 were 25.01 and $13.95 \%$, respectively; Figure 4). This was confirmed by MANOVA, in which significant difference in fungal composition of tarag from Sichuan and Mongolia was detected (Figure 5). Although a few samples overlapped (Figure 4), a MANOVA using the principal components based on the unweighted UniFrac metric further indicated that the difference between the 2 groups was significant $(P=0.046$; Figure 5$)$. Thus, we may conclude that the microbial flora in different samples was stratified by geographic region. The tarag samples used in this study were produced by similar traditionally preserved methods. Therefore, most likely, the microbial structural differences between samples of various regions can be attributed to geographic 

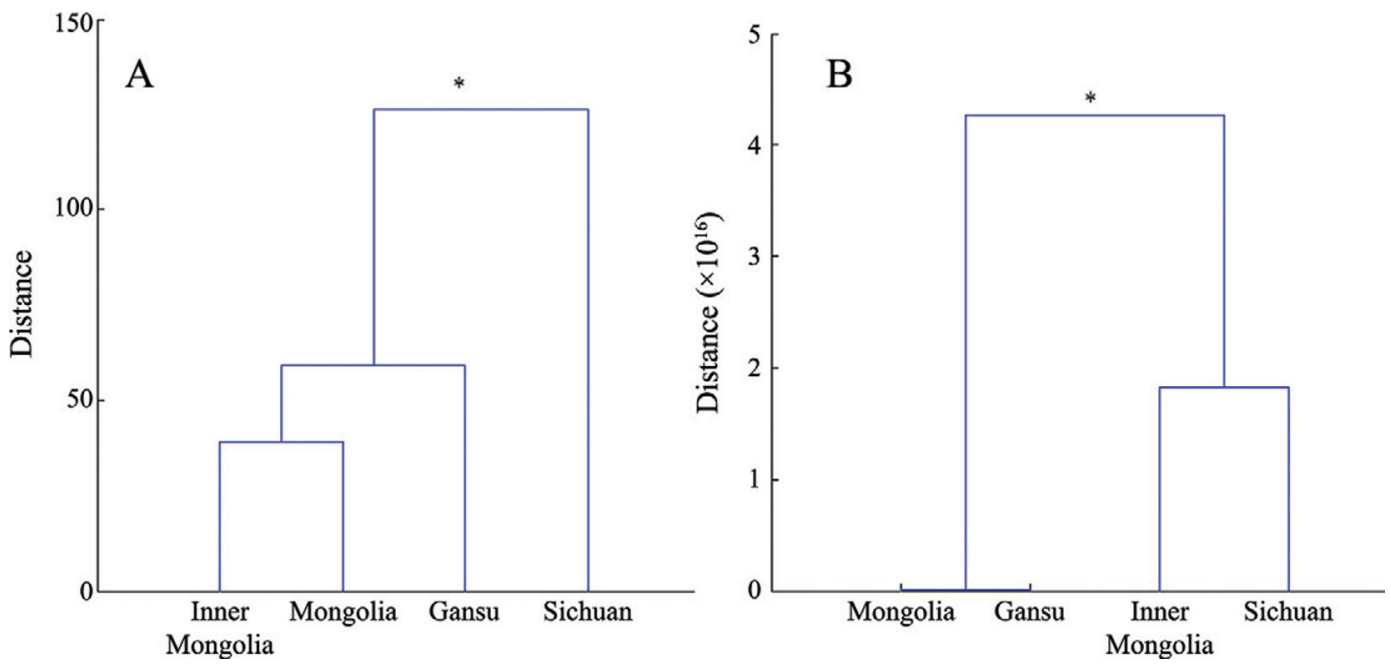

Figure 5. Clustering of gut microbiota based on distances between different groups calculated with multivariate ANOVA of the first 14 principal components of bacterial (A) and fungal (B) diversity data. The Mahalanobis distances between group means are shown. ${ }^{*} P<0.05$. Color version available in the online PDF.

location and other environmental factors. Similarly, Watanabe et al. (2008) reported that the microbial communities of traditional fermented milk varied with the sampling geographic region. Environmental factors, such as elevation, climate, temperature, oxygen level, atmospheric pressure, sunlight, and length of sunlight radiation, may play roles in modulating the microbiome of fermented dairy products (Sun et al., 2010a).

\section{CONCLUSIONS}

This is the first report on the bacterial and fungal communities of Chinese and Mongolian tarag based on a high-throughput pyrosequencing metagenomic approach. To our knowledge, we provided the most thorough description and analysis on the bacterial and fungal communities in tarag sampled in Mongolia and 3 Chinese provinces. Finally, our results strongly suggest that the diversity of microbial flora in different samples was stratified by geographic region.

\section{ACKNOWLEDGMENTS}

This research was supported by the Major State Basic Research Development Program of China (Beijing, China; 973 Program, 2012CB720802), the National Natural Science Foundation of China (Beijing, China; 31025019, 31201396, and 31301518), the National Key Technologies R\&D Program (Beijing, China; 2013BAD18B01), the Special Fund for Agro-Scientific Research in the Public Interest (201303085), and the China Agriculture Research System (Beijing, China; Grant No. CARS-37).

\section{REFERENCES}

Abarenkov, K., R. H. Nilsson, K.-H. Larsson, I. J. Alexander, U. Eberhardt, S. Erland, K. Høiland, R. Kjøller, E. Larsson, T. Pennanen, R. Sen, A. F. S. Taylor, L. Tedersoo, B. M. Ursing, T. Vrålstad, K. Liimatainen, U. Peintner, and U. Kõljalg. 2010. The UNITE database for molecular identification of fungi-Recent updates and future perspectives. New Phytol. 186:281-285.

Airidengcaicike, X. Chen, X. Du, W. Wang, J. Zhang, Z. Sun, W. Liu, L. Li, T. Sun, and H. Zhang. 2010. Isolation and identification of cultivable lactic acid bacteria in traditional fermented milk of Tibet in China. Int. J. Dairy Technol. 63:437-444.

Alegría, Á., P. Szczesny, B. Mayo, J. Bardowski, and M. Kowalczyk. 2012. Biodiversity in Oscypek, a traditional Polish cheese, determined by culture-dependent and -independent approaches. Appl. Environ. Microbiol. 78:1890-1898.

An, Y., Y. Adachi, and Y. Ogawa. 2004. Classification of lactic acid bacteria isolated from chigee and mare milk collected in Inner Mongolia. Anim. Sci. J. 75:245-252.

Bao, Q., J. Yu, W. Liu, M. Qing, W. Wang, X. Chen, F. Wang, M. Li, H. Wang, Q. Lv, and H. Zhang. 2012a. Predominant lactic acid bacteria in traditional fermented yak milk products in the Sichuan Province of China. Dairy Sci. Technol. 92:309-319.

Bao, Q., W. Liu, J. Yu, W. Wang, M. Qing, X. Chen, F. Wang, J. Zhang, W. Zhang, J. Qiao, T. Sun, and H. Zhang. 2012b. Isolation and identification of cultivable lactic acid bacteria in traditional yak milk products of Gansu Province in China. J. Gen. Appl. Microbiol. 58:95-105.

Caporaso, J. G., K. Bittinger, F. D. Bushman, T. Z. DeSantis, G. L. Andersen, and R. Knight. 2010a. PyNAST: A flexible tool for aligning sequences to a template alignment. Bioinformatics $26: 266-267$.

Caporaso, J. G., J. Kuczynski, J. Stombaugh, K. Bittinger, F. D. Bushman, E. K. Costello, N. Fierer, A. G. Pena, J. K. Goodrich, J. I. Gordon, G. A. Huttley, S. T. Kelley, D. Knights, J. E. Koenig, R. E. Ley, C. A. Lozupone, D. McDonald, B. D. Muegge, M. Pirrung, J. Reeder, J. R. Sevinsky, P. J. Turnbaugh, W. A. Walters, J. Widmann, T. Yatsunenko, J. Zaneveld, and R. Knight. 2010b. QIIME allows analysis of high-throughput community sequencing data. Nat. Methods 7:335-336.

Chao, S.-H., H.-Y. Huang, C.-H. Chang, C.-H. Yang, W.-S. Cheng, Y.-H. Kang, K. Watanabe, and Y.-C. Tsai. 2013. Microbial diversity analysis of fermented mung beans (Lu-Doh-Huang) by using pyrosequencing and culture methods. PLoS ONE 8:e63816. 
Chen, H.-C., S.-Y. Wang, and M.-J. Chen. 2008. Microbiological study of lactic acid bacteria in kefir grains by culture-dependent and culture-independent methods. Food Microbiol. 25:492-501.

Dobson, A., O. O'Sullivan, P. D. Cotter, P. Ross, and C. Hill. 2011. High-throughput sequence-based analysis of the bacterial composition of kefir and an associated kefir grain. FEMS Microbiol. Lett. 320:56-62.

Edgar, R. C. 2010. Search and clustering orders of magnitude faster than BLAST. Bioinformatics 26:2460-2461.

El-Baradei, G., A. Delacroix-Buchet, and J.-C. Ogier. 2007. Biodiversity of bacterial ecosystems in traditional Egyptian Domiati cheese. Appl. Environ. Microbiol. 73:1248-1255.

Eliskases-Lechner, F., and W. Ginzinger. 1995. The yeast flora of surface-ripened cheeses. Milchwissenschaft 50:458-462.

Escalante, A., M. Giles-Gómez, G. Hernández, M. S. Córdova-Aguilar, A. López-Munguía, G. Gosset, and F. Bolívar. 2008. Analysis of bacterial community during the fermentation of pulque, a traditional Mexican alcoholic beverage, using a polyphasic approach. Int. J. Food Microbiol. 124:126-134.

Ghannoum, M. A., R. J. Jurevic, P. K. Mukherjee, F. Cui, M. Sikaroodi, A. Naqvi, and P. M. Gillevet. 2010. Characterization of the oral fungal microbiome (mycobiome) in healthy individuals. PLoS Pathog. 6:e1000713.

Hasisurong, Amuguleng, and Manglai.. 2003. Koumiss and its value in medicine. Zhongguo Zhong Yao Za Zhi 28:11-14.

Humblot, C., and J.-P. Guyot. 2009. Pyrosequencing of tagged 16S rRNA gene amplicons for rapid deciphering of the microbiomes of fermented foods such as pearl millet slurries. Appl. Environ. Microbiol. 75:4354-4361.

Huse, S. M., L. Dethlefsen, J. A. Huber, D. Mark Welch, D. A. Relman, and M. L. Sogin. 2008. Exploring microbial diversity and taxonomy using SSU rRNA hypervariable tag sequencing. PLoS Genet. 4:e1000255.

Illeghems, K., L. De Vuyst, Z. Papalexandratou, and S. Weckx. 2012. Phylogenetic analysis of a spontaneous cocoa bean fermentation metagenome reveals new insights into its bacterial and fungal community diversity. PLoS ONE 7:e38040.

Ishii, S., and Y. Konagaya. 2002. Beneficial role of koumiss intake of Mongolian nomads. J. Jpn. Soc. Nutr. Food Sci. 55:281-285.

Jung, J., S. Choi, C. O. Jeon, and W. Park. 2013. Pyrosequencingbased analysis of the bacterial community in Korean traditional seafood, ojingeo jeotgal. J. Microbiol. Biotechnol. 23:1428-1433.

Jung, M.-J., Y.-D. Nam, S. W. Roh, and J.-W. Bae. 2012. Unexpected convergence of fungal and bacterial communities during fermentation of traditional Korean alcoholic beverages inoculated with various natural starters. Food Microbiol. 30:112-123.

Kuczynski, J., J. Stombaugh, W. A. Walters, A. González, J. G. Caporaso, and R. Knight. 2011. Using QIIME to analyze 16S rRNA gene sequences from microbial communities. Curr. Protoc. Bioinformatics 36:10.7.1-10.7.20.

Lattanzi, A., F. Minervini, R. Di Cagno, A. Diviccaro, L. Antonielli, G. Cardinali, S. Cappelle, M. De Angelis, and M. Gobbetti. 2013. The lactic acid bacteria and yeast microbiota of eighteen sourdoughs used for the manufacture of traditional Italian sweet leavened baked goods. Int. J. Food Microbiol. 163:71-79.

Leite, A. M., B. Mayo, C. T. Rachid, R. S. Peixoto, J. T. Silva, V. M. Paschoalin, and S. Delgado. 2012. Assessment of the microbial diversity of Brazilian kefir grains by PCR-DGGE and pyrosequencing analysis. Food Microbiol. 31:215-221.

Letunic, I., and P. Bork. 2007. Interactive Tree Of Life (iTOL): An online tool for phylogenetic tree display and annotation. Bioinformatics 23:127-128.

Li, X.-R., E.-B. Ma, L.-Z. Yan, H. Meng, X.-W. Du, S.-W. Zhang, and Z.-X. Quan. 2011. Bacterial and fungal diversity in the traditional Chinese liquor fermentation process. Int. J. Food Microbiol. 146:31-37.

Liu, W., Q. Bao, Jirimutu, M. Qing, Siriguleng, X. Chen, T. Sun, M. Li, J. Zhang, J. Yu, M. Bilige, T. Sun, and H. Zhang. 2012a. Isolation and identification of lactic acid bacteria from Tarag in Eastern Inner Mongolia of China by 16S rRNA sequences and DGGE analysis. Microbiol. Res. 167:110-115.
Liu, W. J., Z. H. Sun, Y. B. Zhang, C. L. Zhang, Menghebilige, M. Yang, T. S. Sun, Q. H. Bao, W. Chen, and H. P. Zhang. 2012b. A survey of the bacterial composition of kurut from Tibet using a culture-independent approach. J. Dairy Sci. 95:1064-1072.

Lozupone, C., and R. Knight. 2005. UniFrac: A new phylogenetic method for comparing microbial communities. Appl. Environ. Microbiol. 71:8228-8235.

Margulies, M., M. Egholm, W. E. Altman, S. Attiya, J. S. Bader, L. A. Bemben, J. Berka, M. S. Braverman, Y.-J. Chen, Z. Chen, S. B. Dewell, L. Du, J. M. Fierro, X. V. Gomes, B. C. Godwin, W. He, S. Helgesen, C. H. Ho, G. P. Irzyk, S. C. Jando, M. L. I. Alenquer, T. P. Jarvie, K. B. Jirage, J.-B. Kim, J. R. Knight, J. R. Lanza, J. H. Leamon, S. M. Lefkowitz, M. Lei, J. Li, K. L. Lohman, H. Lu, V. B. Makhijani, K. E. McDade, M. P. McKenna, E. W. Myers, E. Nickerson, J. R. Nobile, R. Plant, B. P. Puc, M. T. Ronan, G. T. Roth, G. J. Sarkis, J. F. Simons, J. W. Simpson, M. Srinivasan, K. R. Tartaro, A. Tomasz, K. A. Vogt, G. A. Volkmer, S. H. Wang, Y. Wang, M. P. Weiner, P. Yu, R. F. Begley, and J. M. Rothberg. 2005. Genome sequencing in microfabricated high-density picolitre reactors. Nature 437:376-380.

Masoud, W., M. Takamiya, F. K. Vogensen, S. Lillevang, W. A. AlSoud, S. J. Sørensen, and M. Jakobsen. 2011. Characterization of bacterial populations in Danish raw milk cheeses made with different starter cultures by denaturating gradient gel electrophoresis and pyrosequencing. Int. Dairy J. 21:142-148.

Miyamoto, M., Y. Seto, H. Nakajima, S. Burenjargal, A. Gombojav, S. Demberel, and T. Miyamoto. 2010. Denaturing gradient gel electrophoresis analysis of lactic acid bacteria and yeasts in traditional Mongolian fermented milk. Food Sci. Technol. Res. 16:319-326.

Nam, Y.-D., S.-L. Park, and S.-I. Lim. 2012. Microbial composition of the Korean traditional food "kochujang" analyzed by a massive sequencing technique. J. Food Sci. 77:M250-M256.

Price, M. N., P. S. Dehal, and A. P. Arkin. 2009. FastTree: Computing large minimum evolution trees with profiles instead of a distance matrix. Mol. Biol. Evol. 26:1641-1650.

Prillinger, H., O. Molnár, F. Eliskases-Lechner, and K. Lopandic. 1999. Phenotypic and genotypic identification of yeasts from cheese. Antonie van Leeuwenhoek 75:267-283.

Quigley, L., O. O'Sullivan, T. P. Beresford, R. P. Ross, G. F. Fitzgerald, and P. D. Cotter. 2012. High-throughput sequencing for detection of subpopulations of bacteria not previously associated with artisanal cheeses. Appl. Environ. Microbiol. 78:5717-5723.

Rahman, N., C. Xiaohong, F. Meiqin, and D. Mingsheng. 2009. Characterization of the dominant microflora in naturally fermented camel milk shubat. World J. Microbiol. Biotechnol. 25:1941-1946.

Rogosa, M., J. A. Mitchell, and R. F. Wiseman. 1951. A selective medium for the isolation and enumeration of oral lactobacilli. J. Dent. Res. 30:682-689.

Roh, S. W., K.-H. Kim, Y.-D. Nam, H.-W. Chang, E.-J. Park, and J.-W. Bae. 2010. Investigation of archaeal and bacterial diversity in fermented seafood using barcoded pyrosequencing. ISME J. $4: 1-16$.

Shuangquan, Burentegusi, B. Yu, and T. Miyamoto. 2006. Microflora in traditional starter cultures for fermented milk, hurunge, from Inner Mongolia, China. Anim. Sci. J. 77:235-241

Smith, M. T., A. W. A. M. de Cock, G. A. Poot, and H. Y. Steensma. 1995. Genome comparisons in the yeastlike fungal genus Galactomyces Redhead et Malloch. Int. J. Syst. Bacteriol. 45:826-831.

Sudun, Wulijideligen, K. Arakawa, M. Miyamoto, and T. Miyamoto. 2013. Interaction between lactic acid bacteria and yeasts in airag, an alcoholic fermented milk. Anim. Sci. J. 84:66-74.

Sun, Z., W. Liu, W. Gao, M. Yang, J. Zhang, L. Wu, J. Wang, B. Menghe, T. Sun, and H. Zhang. 2010a. Identification and characterization of the dominant lactic acid bacteria from kurut: The naturally fermented yak milk in Qinghai, China. J. Gen. Appl. Microbiol. 56:1-10.

Sun, Z. H., W. J. Liu, J. C. Zhang, J. Yu, W. Gao, M. Jiri, B. Menghe, T. S. Sun, and H. P. Zhang. 2010b. Identification and characterization of the dominant lactic acid bacteria isolated from traditional fermented milk in Mongolia. Folia Microbiol. (Praha) 55:270-276. 
Tamura, K., D. Peterson, N. Peterson, G. Stecher, M. Nei, and S. Kumar. 2011. MEGA5: Molecular evolutionary genetics analysis using maximum likelihood, evolutionary distance, and maximum parsimony methods. Mol. Biol. Evol. 28:2731-2739.

Van Hoorde, K., T. Verstraete, P. Vandamme, and G. Huys. 2008. Diversity of lactic acid bacteria in two Flemish artisan raw milk Gouda-type cheeses. Food Microbiol. 25:929-935.

Wang, Q., G. M. Garrity, J. M. Tiedje, and J. R. Cole. 2007. Naive Bayesian classifier for rapid assignment of rRNA sequences into the new bacterial taxonomy. Appl. Environ. Microbiol. 73:5261-5267.

Wang, T., G. Cai, Y. Qiu, N. Fei, M. Zhang, X. Pang, W. Jia, S Cai, and L. Zhao. 2012. Structural segregation of gut microbiota between colorectal cancer patients and healthy volunteers. ISME J. $6: 320-329$

Watanabe, K., J. Fujimoto, M. Sasamoto, J. Dugersuren, T. Tumursuh, and S. Demberel. 2008. Diversity of lactic acid bacteria and yeasts in airag and tarag, traditional fermented milk products of Mongolia. World J. Microbiol. Biotechnol. 24:1313-1325.

Welthagen, J. J., and B. C. Viljoen. 1998. Yeast profile in Gouda cheese during processing and ripening. Int. J. Food Microbiol. 41:185-194.

Wyder, M.-T., H.-P. Bachmann, and Z. Puhan. 1999. Role of selected yeasts in cheese ripening: An evaluation in foil wrapped Raclette cheese. Lebensm. Wiss. Technol. 32:333-343.

Yoo, J.-C., J.-B. Sim, H.-K. Kim, H.-S. Chun, and S.-J. Kim. 1994 Purification and properties of membrane-bound alcohol dehydrogenase from Acetobacter sp. HA. Korean J. Microbiol. 32:78-83.

Yu, J., W. H. Wang, B. L. G. Menghe, M. T. Jiri, H. M. Wang, W. J. Liu, Q. H. Bao, Q. Lu, J. C. Zhang, F. Wang, H. Y. Xu, T. S. Sun, and H. P. Zhang. 2011. Diversity of lactic acid bacteria associated with traditional fermented dairy products in Mongolia. J. Dairy Sci. 94:3229-3241. 